\title{
Türkiye için beş yeni kayıt ile Sündiken Dağları (Eskişehir) Limoniidae (Diptera) faunasına katkılar ${ }^{1}$
}
Contributions to the Limoniidae (Diptera) fauna of Sündiken Mountains (Eskişehir), with five new records for Turkey ${ }^{1}$

\author{
Seda B LG N ${ }^{2} \quad$ Okan ÖZGÜL ${ }^{3} \quad$ Hasan KOÇ \\ Summary
}

Fifty one limoniid (Diptera: Nematocera) taxa were collected from Sündiken Mountains of Eskişehir between July 2013 and May 2014. These taxa contained five species new records for Turkish Limoniidae fauna: Eloeophila apicata (Loew, 1871), Eloeophila czernyi (Strobl, 1909) Gonomyia (Gonomyia) lucidula de Meijere 1920, Molophilus (Molophilus) obsoletus Lackshewitz 1940 and Limonia nigropunctata nigropunctata (Schummel, 1829). Distributional data in the study area, Palaearctic distributions and habitats for each new record are given.

Key words: Diptera, Limoniidae, Sündiken, Eskişehir, Turkey, new records

\section{Özet}

Eskişehir'in Sündiken Dağları'ndan Haziran 2013 ve Mayıs 2014 ayları arasında 51 limoniid (Diptera: Nematocera: Limoniidae) taksonu toplanmıştır. Bu taksonlar Türkiye Limoniidae faunası için 5 yeni kayıdı da ihtiva etmektedir: Eloeophila apicata (Loew, 1871), Eloeophila czernyi (Strobl, 1909), Gonomyia (Gonomyia) lucidula de Meijere 1920, Molophilus (Molophilus) obsoletus Lackshewitz 1940 ve Limonia nigropunctata nigropunctata (Schummel,1829). Her bir yeni kayıt için araştırma alanındaki dağılımları, Palaearktik bölgedeki dağılımları ve habitat bilgileri verilmiştir.

Anahtar sözcükler: Diptera, Limoniidae, Sündiken, Eskişehir, Türkiye, yeni kayıtlar

\footnotetext{
1 Bu çalışmanın bir kısmı 23-27 Haziran 2007 tarihinde Eskişehir'de düzenlenen 22. Ulusal Biyoloji Kongresi'nde poster olarak sunulmuş ve sadece özet olarak kongre kitapçığında basıımıştır. Bu çalışma TÜB TAK tarafından desteklenen 113 T039 nolu projenin bir kısmı olup, ilk yazarın yüksek lisans tezinden türetilmiştir.

${ }_{2}^{2}$ Muğla Sıtkı Koçman Üniversitesi, Fen Bilimleri Enstitüsü, Muğla, Türkiye

${ }^{3}$ Muğla Sıtkı Koçman Üniversitesi, Ula Ali Koçman M.Y.O., Ula, Muğla, Türkiye

${ }^{4}$ Muğla Sıtkı Koçman Üniversitesi, Fen Fakültesi, Biyoloji Bölümü, Muğla, Türkiye

* Sorumlu yazar (Corresponding author) e-mail: khasan@mu.edu.tr Alınış (Received): 26.01.2015 Kabul ediliş (Accepted): 03.03.2015
} 


\section{Giriş}

Limoniidae, tür sayısı bakımından Diptera takımının en geniş familyalarından birisidir. Dünya'da 10.547, Palearktik Bölge'de ise 1650 tanımlanmış tür ve alttürle temsil edilmektedir (Oosterbroek, 2015). Türkiye'de ise, önceleri sadece 20 olan limoniid takson sayısı, özellikle 2001'den sonra yerli araştırıcıların araştırmaları sayesinde büyük artış göstererek yüzü aşmıştır.

Limoniidlerin doğadaki yaşam döngüsü kısa bir yumurta evresi, dört larval evre, kısa bir pupa evresi (5-12 gün) ile kısa bir ergin evresinden oluşmaktadır. Limoniidlerin yaşam süreleri de 6 hafta kadar kısa olabileceği gibi, 4 yıl kadar da uzun olabilmektedir (Savchenko et al., 1992). Yaşam süresi tamamen çevresel şartlara, özellikle de sıcaklık ve neme bağlı olarak değişiklik göstermektedir (Reusch \& Oosterbroek, 1997).

Limoniid larvaları çoğunlukla göl ve akarsu kenarındaki bitkiler üzerinde bulunurlar ve çürümüş bitki materyalleri üzerinde, alg ve fungilerle, bazıları da ciğerotları ve yosunlar ile beslenirler. Dicranoptycha, Limonia, Dicranomyia ve bazı Cheilotrichia türlerinin larvaları kuru topraklarda, Orimarga ve bazı Limonia türleri alglerle desteklenmiş nemli köprü kemerlerinde ve uçurum yamaçlarında; Gnophomyia ve Lipsothrix türleri mantar miselleri üzerinde; Metalimnobia türleri de mantarların yenen kısımları üzerinde bulunabilmektedir. Çoğu Limoniinae ve Limnophilinae türleri ise karnivordur (Reusch \& Oosterbroek, 1997). Pupa döneminin hemen öncesinde, larvaları genellikle habitatlarının yakınındaki kuru yerlere doğru hareket ederler (Savchenko, 1989).

Limoniidae erginleri hantal uçtukları için larva ve pupa evresini geçirdikleri alandan çok fazla uzaklaşamazlar. Bu evreleri geçirdikleri habitatların hemen kenarlarındaki tek yıllık otsu bitkiler, çalılıklar ya da orman içlerinde yosunların, otsu bitkilerin ve eğreltilerin oluşturduğu vejetasyonlarda bulunurlar. Halk tarafından sivrisineklere benzetilseler de, ağız parçaları elvermediği için kan ememezler. Erkekleri beslenmezler ve çiftleşmeden hemen sonra ölürler. Dişileri ise bitki özsuları ile beslenirler.

Limoniid ergin ve larvaları kuşların, memelilerin, balıkların ve omurgasızlardan özellikle örümceklerin ve predatör böceklerin besini durumundadır (Brinkmann, 1991; Reusch \& Oosterbroek, 1997; Savchenko et al., 1992).

Tarımsal ürün zararlısı olarak bilinen limoniid larvaları buğday, şeker kamışı, şeker pancarı, yonca, pamuk gibi tarımsal bitkilere, ormanlardaki ağaçların kök ve genç sürgünlerine zarar verirler. Bitkilerin ya da fidanların sürgünlerini toprak üzerinden ya da altından keserek koparırlar (Özgül et al., 2009).

ç Anadolu Bölgesi coğrafik sınırları içinde şimdiye kadar turna sinekleri ile ilgili herhangi bir araştırma yapılmamıştır. Afyonkarahisar, Kütahya ve Uşak illerini içeren Ege Bölgesi'nin ç Batı Bölümü'nde yapılan kapsamlı bir çalışmadan sonra, Türkmen Dağı ile bağlantısı olan Sündiken Dağları'nda yapılacak bu çalışmanın kısmen de olsa tüm Eskişehir ilini temsil edebileceği düşünülmüştür. Ayrıca araştırma bölgesinin Limoniidae faunasının belirlenmesi ve Türkiye faunasına katkılar sağlanması amacıyla bu çalışma gerçekleştirilmiştir.

\section{Materyal ve Yöntem}

Araştırma bölgesi olan Sündiken Dağları, Marmara, ç Anadolu ve Ege Bölgesi arasında zoocoğrafik açıdan bir geçiş bölgesidir. Sündiken Dağları, kuş uçuşu yaklaşık $30 \mathrm{~km}$ eni, $70 \mathrm{~km}$ boyu olan, 210 bin hektarı $\left(2.100 \mathrm{~km}^{2}\right)$ kapsamaktadır. Sündiken Dağları, Eskişehir ilinin yaklaşık \% 17'si kadar bir alana sahip olup, ülkemizin ise \% 0,26'sını oluşturmakla birlikte kuzeyde ve doğuda Sakarya Nehri, güneyde Eskişehir Ovası, batıda ise Bozdağ ile sınırlıdır (Çelik, 2006).

Araştırma alanında birbirlerinden farklı olduğu düşünülen habitatlar seçilmiş ve bu habitatlardan ergin örnekler sıklıkla çapı $40 \mathrm{~cm}$ olan atrap kullanılarak süpürme yöntemiyle ve nadir olarak da akşamları hava kardıktan sonra ışık tuzağı ile toplanmıştır. Kış ayları hariç, Haziran 2014 ve Mayıs 2015 ayları arasında 8 ay yapılan arazi çalışmaları sonucunda Limoniidae familyasına ait 893'ü erkek olmak üzere, 
1106 ergin örnek toplanmıştır. Toplanan bu örnekler ya \% 70 alkol içeren kavanozlar içine, ya da böcek zarflarına alınmışlar ve laboratuara getirilmişlerdir. Bazı örneklerin teşhis karakterlerinin şeffaflaştırıması ve arındırılması amacıyla Dienske (1987)'ye göre preparasyonları yapılmış, sonrasında da daha çok Coe (1950), Dienske (1987), Oosterbroek (2006), Reusch \& Oosterbroek (1997), Podenas et al. (2006) kaynakları kullanılarak teşhisleri sonuçlandırılmıştır. Sınıflandırma için Oosterbroek (2015) takip edilmiştir. Preparasyonları yapılan bu genital kısımlar, içi gliserin ve alkol bulunan küçük kapsüllere alınarak, örneğin geri kalan kısmına iliştirilmiştir. Tespit edilen örnekler Muğla Sıtkı Koçman Üniversitesi, Zooloji Laboratuvarında muhafaza edilmektedir.

\section{Araştırma Sonuçları ve Tartışma}

Bu çalışma, Sündiken Dağları'nda (Eskişehir) 2013 yılı Haziran ve Ekim ayları ile 2014 yılı Mart ve Mayıs ayları arasında toplam 8 ay süren arazi çalışmalarında toplanan örneklere dayanmaktadır. Araştırma alanında çayır sivrisineklerinin bulunabileceği 402 lokalite seçilerek, her bir ayda ortalama 7 gün arazi çalışmaları gerçekleştirilmiştir. Yapılan bu çalışmalarda 891 erkek ve 215 dişi birey olmak üzere toplam 1106 limoniid örneği toplanmıştır. Bu örneklerin yapılan teşhisleri sonucunda 25 cinse ait 51 takson oldukları belirlenmiştir (Çizelge 1).

Çizelge 1. Araştırma alanından tespit edilen limoniid tür ve altürleri, örnek sayıları ve notlar

\begin{tabular}{|c|c|c|}
\hline Tür ve Alttürler & $\begin{array}{l}\text { Örnek } \\
\text { Sayısı }\end{array}$ & Notlar \\
\hline \multicolumn{3}{|l|}{ Altfamilya Chioneinae } \\
\hline 1. Arctoconopa melampodia (Loew, 1873) & 1 & $a, W, E, E S$ \\
\hline 2. Ellipteroides (Ellipteroides) lateralis (Macquart, 1835) & 7 & a, W, ES \\
\hline 3. E. (Protogonomyia) murati Özgül ve Koç, 2014 & 1 & $\mathrm{a}, \mathrm{W}, \mathrm{ES}$, en \\
\hline 4. Erioconopa interposita Stary, 1976 & 1 & $a, W, E S$ \\
\hline 5. Erioptera (Mesocyphona) bivittata (Loew, 1873) & 3 & $\mathrm{a}, \mathrm{W}, \mathrm{E}, \mathrm{ES}$ \\
\hline 6. Erioptera (E.) fusculenta Edwards, 1938 & 283 & $a, e, W, E, E S$ \\
\hline 7. E. (E.) lutea lutea Meigen, 1804 & 25 & $a, e, W, E, E S$ \\
\hline 8. Gonomyia (Gonomyia) lucidula Meijere 1920 & 5 & W, T, ES \\
\hline 9. G. (G.) recta Tonnoir, 1920 & 7 & a, W, ES \\
\hline 10. G. (G.) tenella (Meigen, 1818) & 6 & a, e, W, ES \\
\hline 11. Idiocera (Idiocera) pulchripennis (Loew, 1856) & 10 & $a, e, W, E, E S$ \\
\hline 12. Ilisia inermis Mendl, 1979 & 1 & $\mathrm{a}, \mathrm{W}, \mathrm{ES}$ \\
\hline 13. I. maculata (Meigen, 1804) & 1 & $a, e, W, E S$ \\
\hline 14. Molophilus (Molophilus) appendiculatus (Staeger, 1840) & 3 & $a, e, W, E, E S$ \\
\hline 15. M. (M.) kallemuelleri Mendl, 1984 & 9 & a, W, ES \\
\hline 16. M. (M.) obscurus (Meigen, 1818) & 300 & $a, e, W, E S$ \\
\hline 17. M. (M.) obsoletus Lackschewitz, 1940 & 1 & $\mathrm{~W}, \mathrm{~T}, \mathrm{ES}$ \\
\hline 18. M. (M.) pleuralis Meijere, 1920 & 28 & $a, e, W, E, E S$ \\
\hline 19. M. (M.) propinquus propinquus (Egger, 1863) & 113 & $a, e, W, E, E S$ \\
\hline 20. Symplecta (Psiloconopa) stictica stictica (Meigen, 1818) & 4 & $a, e, W, E, E S$ \\
\hline 21. S. (Symplecta) hybrida (Meigen, 1804) & 13 & $a, e, W, E, N, O, E S$ \\
\hline 22. S. (Trimicra) pilipes (Fabricius, 1787) & 1 & $a, e, W, E, N, O, N e, A f, A v, A O, E S$ \\
\hline 23. Tasiocera (Dasymolophilus) murina (Meigen, 1818) & 1 & $a, W, E S$ \\
\hline
\end{tabular}


Çizelge 1. (Devamı)

\section{Altfamilya Limnophilinae}

24. Afrolimnophila minima (Savchenko, 1971)

25. Austrolimnophila (A.) brevicellula Stary, 1977

26. Dicranophragma (Brachylimnophila) adjunctum (Walker, 1848)

27. Eloeophila apicata (Loew, 1871)

28. E. czernyi (Strobl, 1909)

29. E. maculata (Meigen, 1804)

30. Euphylidorea phaeostigma (Schummel, 1829)

31. Hexatoma (Eriocera) chirothecata (Scopoli, 1763)

32. Limnophila (Limnophila) schranki Oosterbroek, 1992

33. Paradelphomyia (Oxyrhiza) senilis (Haliday, 1833)

34. Phylidorea (Phylidorea) ferruginea (Meigen, 1818)

35. Pilaria discicollis (Meigen, 1818)

36. P. fuscipennis (Meigen, 1818)

37. P. scutellata (Staeger, 1840)

38. Pseudolimnophila (Pseudolimnophila) sepium (Verrall, 1886)

\section{Altfamilya Limoniinae}

39. Dicranomyia (Dicranomyia) didyma (Meigen, 1804)

40. D. (D.) goritiensis (Mik, 1864)

41. D. (D.) lucida Meijere 1918

42. D. (D.) mitis (Meigen, 1830)

43. D. (D.) modesta (Meigen, 1818)

44. D. (Glochina) sericata (Meigen, 1830)

45. Dicranoptycha fuscescens (Schummel, 1829)

46. Limonia macrostigma (Schummel, 1829)

47. L. nigropunctata nigropunctata (Schummel,1829)

48. L. nubeculosa Meigen 1804

49. L. pannonica (Kowarz, 1868)

50. L. phragmitidis (Schrank, 1781)

51. Orimarga (Orimarga) attautena Özgül \& Koç 2014
6

$a, W, E S$

11

a, W, ES

a, e, W, ES

W, E, T, ES

W, T, ES

a, e, W, E, ES

a, W, ES

a, e, W, ES

a, e, W, E, ES

$a, e, W, E, E S$

a, e, W, E, ES

a, e, W, ES

a, e, W, E, ES

a, W, E, ES

a, e, W, E, ES

a, W, E, ES

a, W, ES

a, W, ES

a, e, W, E, ES

a, e, W, E, N, ES

a, e, W, ES

a, e, W, E, ES

a, e, W, E, O, ES

W, T, ES

10

a, e, W, E, N, ES

28

a, e, W, ES

23

a, e, W, E, ES

17

a, W, ES, en

*a: Türkiye'nin Asya kısmı, e: Türkiye'nin Avrupa kısmı, W: Batı Palearktik, E: Doğu Palearktik, N: Nearktik,

O: Oriental, Nt: Neotropik, Af: Afrotropik, AO: Australian/Oceanian, T: Türkiye faunası için yeni kayıt,

S: ES: Araştırma alanı faunası için yeni kayıt, en: endemik. 


\section{Gonomyia (Gonomyia) lucidula Meijere 1920}

ncelenen materyal ve lokalite: Eskişehir, Alpu, Otluk-Karacaören Yolu 5. km (400 $00^{\prime} 27^{\prime \prime} \mathrm{N} / 31^{\circ}$

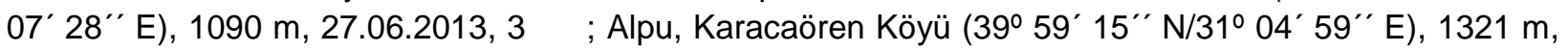
27.06.2013, $10^{x}$.

Habitat: Çam ormanı içindeki dere kenarında bulunan ısırgan, nane, yabani çilek, orkide, böğürtlenler üzerinden.

Palearktik bölgedeki yayılışı: Avusturya, Belçika, Bulgaristan, Hırvatistan, Çek Cumhuriyeti, Danimarka, Estonya, Fransa, Almanya, Büyük Britanya, rlanda, talya, Letonya, Litvanya, Lüksemburg, Hollanda, Norveç, Polonya, Romanya, Slovakya, sveç, sviçre, Ukrayna, Rusya, Kuzey Kafkasya, Gürcistan, Ermenistan, Azerbaycan (Oosterbroek, 2015).

\section{Molophilus (Molophilus) obsoletus Lackschewitz, 1940}

ncelenen materyal ve lokalite: Eskişehir, Alpu, Arıkaya-Sündiken Tepesi Arası (39ํ5' 06" N/31을 04' 50" E), $1628 \mathrm{~m}, 27.06 .2013,10^{*}$.

Habitat: Çam ormanı içindeki dağ çeşmesi etrafındaki tek yıllık otsu bitkiler.

Palearktik bölgedeki yayılışı: Bulgaristan, Romanya (Oosterbroek, 2015).

\section{Eloeophila apicata (Loew, 1871)}

ncelenen materyal ve lokalite: Eskişehir, Merkez, Şifalı Su yolu (39 $\left.58^{\prime} 15^{\prime \prime} \mathrm{N} / 30^{\circ} 45^{\prime} 38^{\prime \prime} \mathrm{E}\right)$, $1217 \mathrm{~m}, 17.07 .2013,3 \mathrm{o}^{\pi} \mathrm{o}^{\pi}$.

Habitat: Çam ormanı içindeki akarsu kenarında tek yıllık otsu bitkiler üzerinden, demir otu ve yabani nane ağırlıklı.

Palearktik bölgedeki yayılışı: Avusturya, Bosna-Hersek, Bulgaristan, Hırvatistan, Çek Cumhuriyeti, Finlandiya, Fransa, Almanya, ngiltere, Yunanistan, Macaristan, rlanda, talya, Litvanya, Polonya, Romanya, Slovakya, Slovenya, spanya, sviçre, Ukrayna, Gürcistan, ran, Türkmenistan (Oosterbroek, 2015).

\section{Eloeophila czernyi (Strobl, 1909)}

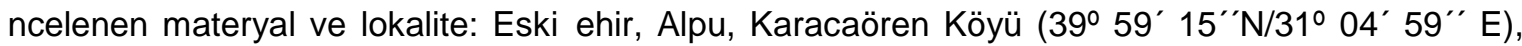
1321 m, 27.06.2013, 2 우 우 ; Merkez, Sakarılılıca-Eskişehir Yolu 20. km (400’ 23” N/30 40’ 17” E), 453 $\mathrm{m}, 23.09 .2013,2 \sigma^{x} \sigma^{x}$.

Habitat: : Çam ve meşe ağaçlarından oluşan orman içindeki sızıntı şeklinde çok az akan dere kenarında bulunan tek yıllık otsu bitkiler ve çalılıklar üzerinden, böğürtlen ve nane ağırlıklı.

Palearktik bölgedeki yayılışı: Fransa, talya, spanya (Oosterbroek, 2015).

\section{Limonia nigropunctata nigropunctata (Schummel,1829)}

ncelenen materyal ve lokalite: Eskişehir, Merkez, Çalkara Köyü (39 58’ 59” N / 30 23’ 38” E), $981 \mathrm{~m}, 24.04 .2014,2 \sigma^{\pi} \sigma^{\top}$.

Habitat: Kenarında kavak ağaçları bulunan dere kenarı, ısırgan otu ağırlıklı tek yıllık otsu bitkiler üzerinden. 
Palearktik bölgedeki yayılışı: Arnavutluk, Avusturya, Belçika, Bosna-Hersek, Bulgaristan, Hırvatistan, Çek Cum., Danimarka, Fransa, Almanya, Büyük Britanya, Macaristan, talya, Litvanya, Lüksemburg, Makedonya, Karadağ, Hollanda, Polonya, Romanya, Sırbistan, Slovakya, Slovenya, sveç, sviçre, Ukrayna, Rusya (Oosterbroek, 2015).

Sündiken Dağları'nda (Eskişehir) gerçekleştirilen bu çalışmada belirlenen 51 limoniid taksonundan 5’i ülkemiz Limoniidae (Diptera) faunası için ilk kez kaydedilmektedir. Bunlardan Eloeophila apicata (Loew, 1871) hem Batı hem de Doğu Palearktik dağılışlı iken, geri kalan 4'ü sadece Batı Palearktik dağılışıdır. Gonomyia (Gonomyia) lucidula de Meijere 1920 ve Eloeophila apicata (Loew, 1871) Batı Palearktik bölgede yaygın bir şekilde bulunurken, Molophilus (Molophilus) obsoletus Lackschewitz, 1940'ın yeryüzünden tespit edildiği 3. ülke ve ayrıca Eloeophila czernyi (Strobl, 1909)'un yeryüzünden tespit edildiği 4. ülke Türkiye olmaktadır.

Bu çalışmayla Akdeniz'de bulunan 3 adadan (Korsika, Sardunya ve Sicilya) tespit edilen Eloeophila czernyi (Strobl, 1909)'nin Palearktik bölgede yaşadığı en doğudaki dağılış sınırı ve ayrıca daha önce Bulgaristan ve Romanya'dan tespit edilmiş olan Molophilus (Molophilus) obsoletus Lackschewitz, 1940'ın Palearktik bölgede yaşadığı coğrafik alanın hem en doğudaki hem de en kuzeydeki dağılış sınırı Eskişehir-Sündiken Dağları olarak güncellenmiştir. Bu çalışma, bu iki türe Avrupa'nın tüm güney-doğu kısımlarında da önümüzdeki yıllarda rastlanılabileceği intimalinin yüksek olduğunu bize göstermektedir. Ayrıca bu çalışmayla, Paleartik bölgede geniş dağılımlı olan Gonomyia (Gonomyia) lucidula de Meijere 1920'nin bu bölgedeki en güney sınırı Eskişehir-Sündiken Dağları olarak genişlemiştir.

Eskişehir Sündiken Dağlarından tespit edilen ve daha önce Aydın ilinden kaydı verilmiş Arctoconopa melampodia (Loew, 1873); daha önce Kütahya ilinden yeni kayıt olarak verilmiş türler olan Erioconopa interposita Stary, 1976, Gonomyia (Gonomyia) recta, Molophilus (Molophilus) appendiculatus (Staeger, 1840) ve Tasiocera (Dasymolophilus) murina (Meigen, 1818); Afyonkarahisar'dan bilinenEuphylidorea phaeostigma (Schummel, 1829) ve Uşak'tan bilinen Limnophila (Limnophila) schranki Oosterbroek, 1992 bu çalışmayla birlikte Türkiye'den 2. kez kaydedilmektedir.

Limonia nigropunctata nigropunctata (Schummel,1829) ise, Palearktik bölgede Rusya'nın kuzeydoğusu (Rusya'nın Avrupa kısmı) ve Ukrayna'ya ek olarak Avrupa'nın orta ve kuzey bölgelerinde dağılıs göstermektedir. Genellikle Palearktik bölgede 940 - 1500 m'ler arasından toplanmış olan bu taksona ait örnekler, Sündiken Dağları'nın kuzey kesiminde 960 m'den tespit edilmiştir. klim şartlarının sert olduğu ve yüksekliğin fazla olduğu bölgelere has bir takson olduğu düşünülmektedir. Bu yüzden de henüz Avrupa'nın Akdeniz kıyılarından tespit edilememiştir.

Tespit edilen türlerden Orimarga (Orimarga) attautena Afyonkarahisar ve Kutahya'dan, Ellipteroides (Protogonomyia) murati ise Kütahya'dan tanımlanmıştır (Özgül \& Koç, 2014). Türkiye için endemik olan söz konusu türler Sündiken Dağları'ndan 2. kez kaydedilmektedir.

Türkiye'de limoniidae tür ve alttürlerinin araştırıldığı diğer iller ile limoniid tür kompozisyonu karşılaştırıldığında araştırma bölgesi en çok Kütahya'ya (\% 70), daha sonra da Afyonkarahisar’a (\% 62) benzemektedir. Eskişehir sınırları içerisinde yer alan Türkmen Dağı'nın doğu yamaçlarının Kütahya il sınırları içerisinde yer almasına ek olarak, Sündiken Dağları içinde yer alan Bozdağ'ın Türkmen Dağı ile bağlantılı olması ve türlerin geçişi için bir güzergah oluşturması, Sündiken Dağları ile Kütahya illerinden tespit edilen türlerin birbirine benzerlik oranlarını arttırdığı düşünülmektedir.

Her ne kadar yüzölçümü olarak Türkiye'nin \% 0.26'sı kadar küçük bir alana sahip olsa da, Türkiye'den bu çalışmayla birlikte belirlenen limoniid tür ve alttürlerin toplam sayısının \% 36 'sı sadece Sündiken Dağları'ndan tespit edilmiş bulunmaktadır. Bu sonuçlar, birçok iklimsel ve coğrafik çeşitllikleri bünyesinde barındıran ülkemizden çok daha fazla limoniid tür ve alttürlerinin tespit edilebileceğinin açık bir göstergesidir. 


\section{Teşekkür}

Yazarlar finansal desteğinden ötürü TÜB TAK’a teşekkür ederler (Proje no: 113T039).

\section{Yararlanılan Kaynaklar}

Brinkmann, R. 1991. Zur habitatpräferenz und phänologie der Limoniidae, Tipulidae und Cylindrotomidae (Diptera) im Bereich eines norddeutschen Tieflandbaches, Faun.-Ökol. Mitt. Suppl., 11: 1-156.

Coe, R.L. 1950. Family Tipulidae. Handbooks for the Identification of British Insects 9: 1-66.

Çelik, N. 2006. Sündiken Dağları Kütlesi'nin Yetişme Ortamı Özellikleri ve Sınıflandırılması. Ü Fen Bilimleri Enstitüsü (Basılmamış) Doktora Tezi, stanbul, $310 \mathrm{~s}$.

Dienske, J. W. 1987. An illustrated Key to the Genera and Subgenera of the Western Palaearctic Limoniidae (Insecta: Diptera), including a Description of the External Morphology, Stutg. Beitr. Naturk. (A), 409: 1-52.

Oosterbroek, P. 2006. The European families of the Diptera, identification, diagnosis, biology. Knnv-Uitgeverij, Utrecht, $205 \mathrm{p}$.

Oosterbroek, P. 2015. Catalogue of the craneflies of the World (Insecta, Diptera, Nematocera, Tipuloidea) available from: http://ip30.eti.uva.nl/ccw (Date accessed: 03 Jan).

Özgül, O. \& H. Koç, 2014. Four new species of Limoniidae (Diptera, Nematocera) from the Inner-West Anatolian subregion of Turkey. Florida Entomologist, 97: 620-625.

Özgül, O., H. Koç, M., Barlas \& A. Tonguç, 2009. Güneybatı Anadolu Bölgesi Limoniidae (Diptera, Nematocera) faunası. S. Ü. Fen Fak. Derg., 33: 61-66.

Podenas, S., W. Geiger, J. P. Haenni \& Y. Gonseth, 2006. "Limoniidae ve Pediciidae de Suisse, 1-375”. In: Fauna Helvetica: 14, Schweizerische Entomologische, Gesellschaft, 375 p.

Reusch, H. \& P. Oosterbroek, 1997. "Diptera, Limoniidae and Pedicidae, Short-palped Crane Flies, 105-132". In: Aquatic insects of North Europe- A Taxonomic Handbook (Ed: A. N. Nilsson). 2. Apollo Books, Stenstrup, 440 p.

Savchenko, E., N. P. Oosterbroek \& J. Starý, 1992. "Family Limoniidae 183-369". In: Catalogue of Palaearctic Diptera, (Eds: A. Soos, L. Papp, \& P. Oosterbroek), Vol: 1, Hungarian Natural History Museum, Budapest, 520 p.

Savchenko, E. N. 1989. "Family Tipulidae, 75-118". In: Keys to the Insects of the European Part of the USSR (Ed: G.A. Bei-Bienko), Vol: 5, 81, Leningrad, 1233 p. 